\title{
The influence of anesthetics on substantia nigra tyrosine hydroxylase expression and tau phosphorylation in the hypoxic-ischemic near-term lamb
}

\author{
Melinda Barkhuizen ${ }^{1,4}$, Fleur J P van Dijck ${ }^{1,4}$, Reint K Jellema ${ }^{1}$, Ruth Gussenhoven ${ }^{1}$, Imke Engelbertink ${ }^{1}$, \\ Ralph van Mechelen ${ }^{1}$, Jack P M Cleutjens ${ }^{3}$, Matthias Seehase ${ }^{1}$, Harry W M Steinbusch ${ }^{2}$, Luc J Zimmermann ${ }^{1}$, \\ A W Danilo Gavilanes ${ }^{1}$ and Boris W Kramer $^{1}$
}

BACKGROUND: General anesthetics could protect key neurotransmitter systems, such as the dopaminergic system, from hypoxic-ischemic encephalopathy (HIE) by limiting excessive glutamatergic neurotransmission. However, anesthetics may adversely affect inflammation and tau phosphorylation.

METHODS: A near-term sheep model of HIE by umbilical cord occlusion (UCO) under anesthesia was used. The effect of propofol and isoflurane on the dopaminergic neurotransmitter phenotype in the substantia nigra (SN) was studied using tyrosine hydroxylase immunohistochemistry. The overall microglial response and tau phosphorylation were also measured in the $\mathrm{SN}$, surrounding the midbrain gray matter structures and the hippocampal white matter.

RESULTS: The isoflurane-treated UCO group had fewer tyrosine hydroxylase-expressing neurons in the $\mathrm{SN}$ at $8 \mathrm{~h}$ after the insult than the propofol-treated UCO or sham-operated groups $(P<0.05)$. The microglial response was unchanged in the $\mathrm{SN}$ region. In the thalamus and the hippocampal stratum moleculare layer, the propofol-treated UCO group had a lower microglial response than the corresponding sham-operated group. Both UCO and the use of anesthetics additively increased tau phosphorylation in the SN region, thalamus, and hippocampus.

CONCLUSION: The choice of anesthetics is important for an emergency C-section. Propofol could potentially protect the dopaminergic neurotransmitter phenotype within the SN at the cost of a widespread increase in tau phosphorylation.

$\mathbf{H}$ ypoxic-ischemic encephalopathy (HIE) remains an important cause of life-long disabilities, such as cerebral palsy, learning difficulties, and attention-deficit hyperactivity disorder (ADHD) $(1,2)$. Damage to the substantia nigra (SN) pars compacta could partially explain the motor and behavioral deficits after HIE in both term and preterm infants, and has been reported in post-mortem examinations of human infants with severe HIE from 32.5 weeks of gestation to term (3). The nigrostriatal dopaminergic tract is an important mediator of movement control. This tract is frequently affected in the rodent models of HIE at most gestational ages (GAs), ranging from the equivalent of the extreme preterm human brain at 28 weeks of gestation (4) to the equivalent full-term human brain (5). In humans, SN abnormalities have also been associated with ADHD (6).

Neuron loss after HIE can occur in several phases. Immediately after the insult, high-energy metabolites are depleted, which leads to neuronal depolarization, increased glutamate release, and reduced glutamate reuptake by astrocytes. This results in an accumulation of excitatory amino acids outside the cell. Many neurons recover from the initial injury, only to deteriorate during the second phase of cerebral energy failure at $6-15 \mathrm{~h}$ after HIE in humans and at $5-48 \mathrm{~h}$ in sheep $(7,8)$. The second phase of the injury leads to microglial activation, which increases the inflammatory cytokine production and worsens the neurological injury (9). The dopaminergic neurotransmitter system is especially sensitive to the combined effects of excessive glutamate release and microglial-mediated inflammation. The therapies that prevent this may be neuroprotective $(4,7)$.

Several general anesthetics could potentially reduce excitotoxicity by antagonizing the glutamatergic $\alpha$-amino-3hydroxy-5-methyl-4-isoxazolepropionic acid (AMPA) and $N$-Methyl- $D$-aspartate (NMDA) receptors to various degrees (10). Although anesthetic exposure is thought to be toxic to the healthy developing brain, by increasing apoptosis and disrupting neurogenesis (11), in the context of an ischemic insult, anesthetics may be neuroprotective by activating the pro-survival pathways, and reducing excitotoxicity, apoptosis, and inflammation (12). The anti-inflammatory effects vary among anesthetics, with studies suggesting that inhalation anesthetics enhances the microglial cytokine release, whereas propofol suppresses the cytokine release (13), by reducing the

\footnotetext{
Department of Pediatrics, Maastricht University Medical Center, Maastricht, The Netherlands; ${ }^{2}$ Department of Neuroscience, School for Mental Health and Neuroscience, Maastricht University, Maastricht, The Netherlands; ${ }^{3}$ Department of Pathology, CARIM, Maastricht University, Maastricht, The Netherlands. Correspondence: Boris W. Kramer (b.kramer@mumc.nl)

${ }^{4}$ The first two authors contributed equally to this work.

Received 20 September 2017; accepted 22 February 2018; advance online publication 9 May 2018. doi:10.1038/pr.2018.42
} 
microglial activation after brain injury (14). We developed a lamb model, at $89 \%$ of gestation, in which anesthetic administration to the ewe before emergency C-section acts as an early treatment to the near-term fetus in distress due to umbilical cord occlusion (UCO), which causes a severe acute hypoxic-ischemic insult. The anesthetic administration is continued in the newborn lamb post delivery as an early postinsult therapy (15-17). We investigated the use of propofol and isoflurane in this scenario, as they are both rapid-acting anesthetics that can be administered during an emergency C-section, which cross the placenta (18), and both anesthetics inhibit glutamatergic AMPA and NMDA receptors (10). We have previously observed several neuroprotective effects of propofol anesthesia in this model, including functional improvements of the auditory pathway (17) and reduced seizure activity (16). Propofol also had molecular benefits such as reduced levels of pro-apoptotic caspases, increased levels of anti-apoptotic proteins, less reactive oxygen species, less mitochondrial damage, and reduced NMDA receptor expression in the brain (16).

Unfortunately, general anesthetics are also known to cause aberrant tau phosphorylation, which is thought to exacerbate the presentation of neurodegenerative diseases (19). Soluble tau is a microtubule-associated protein, which modulates the stability of axonal microtubules to allow the formation of axons and dendrites during normal neurological development. Translocation of the tau from the axons and posttranslational modifications, such as hyperphosphorylation, disrupt tau's microtubule-stabilizing functions and are associated with neuropathology $(20,21)$. After hypoxic or traumatic injuries, the neuronal tau is released extracellularly, and can function as a general biomarker of axonal injury. Tau is important in the neonatal setting, and elevated serum or cerebrospinal fluid tau levels have been linked to worse outcomes after several neonatal encephalopathies, including HIE $(22,23)$. The primary aim of this study was to determine whether propofol and isoflurane could maintain the dopaminergic neurotransmission by preventing a reduction in tyrosine hydroxylase-expressing neurons in the $\mathrm{SN}$ after $\mathrm{UCO}$, as this could potentially reduce the long-term motor morbidity and attention deficits $(4,24)$. The secondary objectives of this study were to determine the effect of anesthesia therapy on microglial response and tau hyperphosphorylation after UCO, as these factors are known to contribute to SN neuronal loss $(25,26)$. The final objective of this study was to determine whether the changes in microglial response and tau phosphorylation were limited to the $\mathrm{SN}$ or widespread throughout the susceptible regions. For this, we extended our investigation of microglia and tau to other regions known to be vulnerable to hypoxic ischemia, such as the hippocampus, fornix, and thalamus $(27,28)$.

\section{METHODS}

\section{Study Design}

The study was approved by the Animal Research Ethics Committee, Maastricht University, the Netherlands. Care was taken to minimize the number of sheep used and to minimize the discomfort of the sheep, in accordance with the EU Directive 2010/63/EU for animal experiments. The study design was described in detail previously (15-17). In brief, the date-mated Texel ewes and their 23 singleton near-term fetuses of both sexes at a mean GA of 133-134 days (89\% of gestation) were used in the experimental protocol. The ewes were randomized for the allocation of sedation with either propofol or isoflurane, which was continued in the near-term lamb after birth. This made blinded administration of the drug prohibitive. The fetuses were randomized for the induction of global hypoxiaischemia by UCO or a sham procedure. The final group sizes were as follows: six UCO-isoflurane (UCO-I), six UCO-propofol (UCO-P), five sham isoflurane (Sh-I), and six sham propofol (Sh-P). An additional four lambs were killed directly after near-term delivery and were served as GA controls $(15,16)$.

\section{Induction of UCO and Sedation}

The experimental protocol used in this study and the clinical outcomes of the lambs have been published previously $(15,16)$. Briefly, the pregnant ewes were intubated and randomized to receive general anesthesia with either isoflurane $(1-2 \%)$ or propofol $(25 \mathrm{mg} /$ $\mathrm{kg} / \mathrm{h}$ ) during C-section. Both anesthesia types were supplemented by continuous remifentanil infusion $(3 \mu \mathrm{g} / \mathrm{kg} / \mathrm{min})$. The fetus was born through a small incision in the uterus, instrumented with an endotracheal tube, and catheters in the femoral artery, umbilical artery, and external jugular vein. The umbilical cord was gently extracted through the incision of the uterus and occluded with a vascular occluder until the mean arterial blood pressure dropped below $30 \mathrm{~mm} \mathrm{Hg}$. From that time point onward, the occlusion was continued for exactly 2 min (refs 15,16). After the end of the occlusion, the lambs were taken out and given $20 \mathrm{~s}$ during $\mathrm{C}$-section to recover spontaneously while experiencing tactile stimulation. The lambs were initially ventilated with a resuscitator bag and resuscitated with heart massage during their transport to an incubator (IW930 Series CosyCot ${ }^{\mathrm{TM}}$ Infant Warmer, Fisher \& Paykel Healthcare, Berkshire, UK), where the lambs were connected to a ventilator Servo 900C (Siemens-Elema, Solna, Sweden) and put into plastic foils to prevent cooling effects. The incubator constantly maintained the lamb's core temperature of $38-38.5^{\circ} \mathrm{C}$, as controlled with a rectal thermometer. Chest compression was continued until the heart rate was $>80$ beats $/ \mathrm{min}$. Adrenaline was administered in augmenting doses from 30 to $100 \mu \mathrm{g}$. A volume bolus of ringer lactate of $10 \mathrm{ml} / \mathrm{kg}$ body weight was administered after the first dose of adrenaline. The sedation was maintained either with isoflurane $(0.5-1.0 \%)$ or propofol $(1-3 \mathrm{mg} / \mathrm{kg} / \mathrm{h})$, according to maternal treatment, was and supplemented with remifentanil $(3 \mu \mathrm{g} / \mathrm{kg} / \mathrm{min})$ in both the groups. The sham-treated fetuses had the same instrumentation and sedation as the UCO groups, without occlusion of the umbilical cord. At $8 \mathrm{~h}$ after delivery, the lambs were killed using an intravenous injection of T61 (Veterinaria AG, Zürich, Switzerland) $(15,16)$.

\section{Immunohistochemistry}

After killing the lambs, the fetal brain was removed from the skull. The right hemisphere was submersion-fixed in ice-cold $4 \%$ paraformaldehyde for 3 months, and was subsequently stored in phosphate-buffered saline with sodium azide until being embedded in gelatin, when it was placed in $4 \%$ paraformaldehyde for an additional $2 \mathrm{~h}$. The serial coronal sections $(50 \mu \mathrm{m})$ were cut on a Leica VT 1200 S vibrating microtome (Leica Biosystems, Nussloch, Germany). The free floating sections from the level of mid-thalamus and posterior hippocampus to the SN were used in the immunohistochemical analyses. To detect and quantify dopamine-producing neurons, we conducted anti-tyrosine hydroxylase $(\mathrm{TH})$ immunohistochemistry on every tenth slide. Antigen was retrieved on the free-floating sections with incubation in $0.1 \mathrm{M}$ citrate buffer at $60^{\circ} \mathrm{C}$ for $20 \mathrm{~min}$, followed by incubation with a mouse-monoclonal anti-TH antibody (1:800, Immunostar, Hudson, WI, cat. ID. 22941) for 3 days. The slides were then incubated with donkey 
anti-mouse-biotin (1:400; Jackson Immunoresearch, Suffolk, UK), followed by incubation with the Avidin-Biotin-Complex (ABC, 1:800 Vector, Peterborough, UK). Staining was visualized with a 3,3'diaminobenzidine solution (DAB). Microglia was detected in an adjacent series, of every tenth section, using immunohistochemistry against anti-ionized calcium-binding-adapter molecule 1 . The slices were incubated overnight with a monoclonal rabbit-anti-ionized calcium-binding-adapter molecule 1-primary antibody (1:1,000, WAKO Chemicals, Nuss, Germany, cat. 019-19741), followed by incubation with donkey anti-rabbit-biotin (1:800; Jackson Immunoresearch) and $\mathrm{ABC}$ (1:800; Vector). The staining was visualized with $\mathrm{DAB}$ using nickel intensification ( $0.2 \%$; DAB-Ni). Tau phosphorylation was detected in additional series of every tenth section. The sections were pretreated with $98 \%$ formic acid for 20 min for antigen retrieval and incubated with mouse-monoclonal primary antibodies against various stages of tau phosphorylation seen in tauopathies, such as Alzheimer's disease. We measured early tau phosphorylation with an anti-phosphorylated Ser-202-tau antibody (CP13, 1:100, a generous gift from Peter Davies, Albert Einstein College of Medicine, New York, NY), and late-stage tau phosphorylation with an antibody against phosphorylated Ser396/Ser404-tau (PHF-1, 1:100, Dr. Davies). We also used an antibody against early conformational changes in tau (MC1, 1:100, Dr. Davies). This was followed by incubation with donkey anti-mouse-biotin (1:400; Jackson Immunoresearch), followed by ABC (1:800; Vector) and DAB visualization. The stained sections were mounted on glass slides, embedded using Depex (Merck, Amsterdam-Zuid, the Netherlands), cover-slipped, and photographed. The coverslips on the TH slides were removed for counterstaining with Nissl solution $(0.1 \%$ cresyl violet acetate) for $40 \mathrm{~min}$ to identify the non-TH-expressing neurons. The hippocampus from an aged transgenic Alzheimer disease mouse (E4FAD) (29) and a post-mortem Alzheimer's disease patient (a generous gift from Fred van Leeuwen) was used as a positive control for tau immunohistochemistry.

\section{Quantitative and Qualitative Analyses}

All the analyses were conducted by a blinded investigator. Four slides per lamb on the same Bregma level were used for each analysis. For the analysis of IBA1 and tau immunoreactivity, digital images of the hippocampal CA3 and DG layers (tau) and white matter layers adjacent to these layers (IBA-1), fornix, thalamus, and midbrain region surrounding the $\mathrm{SN}$ were taken at $\times 2, \times 10$, and $\times 20$ magnification using an Olympus DP 70 digital camera attached to an Olympus AX 70 microscope with Cell-P software Version 2.3 (Soft Imaging System, Münster, Germany). The position of the SN was determined by overlaying the TH-positive slides. The $\times 20$ magnification images were used for the ionized calcium-bindingadapter molecule 1 analysis. The area fraction of IBA1 immunoreactivity was determined with a standard intensity threshold to determine the positive staining using Image 1.5 software (US National Institutes of Health, Bethesda, MD). For tau phosphorylation, images taken at $\times 10$ magnification were scored according to the coloring intensity ranging from 1 to 4 .

For the TH analysis, photos of the $\mathrm{SN}$ were taken at $\times 5$ magnification with a Leica DM3000 microscope, and the TH-positive cells were counted using the Leica QWin software (Leica Microsystems, Cambridge, UK), and were expressed as the total cell count over the four slides in the SN and as densities in cells $/ \mathrm{mm}^{2}$. After the Nissl counterstain, photos were taken at $\times 10$ magnification, and the amount of the TH-labeled and non-labeled neurons were counted to calculate the percentage of non-labeled neurons within the SN boundaries per slice.

\section{Data Analysis}

A statistical analysis was performed with the Stata10 software package (Statacorp, College Station, TX). All the data were assessed with the non-parametric Kruskal-Wallis test with post hoc Dunn's analysis. The Bonferroni correction for multiple testing was applied to the microglia and phosphorylated-tau immunohistochemistry, where multiple regions were investigated. Statistical significance was defined as two-tailed $P$ values $<0.05$. The results are shown as average \pm S.E.M. Graphs were made with GraphPad Prism 6 (GraphPad, La Jolla, CA).

\section{RESULTS}

\section{The UCO Procedure and General Characterization}

The total duration required for UCO was $10.7 \pm 0.3 \mathrm{~min}$ in the propofol-treated lambs and $11.3 \pm 0.6 \mathrm{~min}$ in the isofluranetreated lambs ( $P=$ not significant), as previously described $(15,16)$. All the UCO animals developed bradycardia (heart rate $<30$ beats/min), and all, except one UCO-propofol lamb, required resuscitation. All the lambs survived the insult, and the birth weights were comparable between groups $(15,16)$. In a previous study, we also described the ongoing seizure activity in all lambs that underwent UCO, despite the sedation (16). The combination of cardiac arrest, in all but one UCO lamb, and seizure activity is consistent with the clinical picture of a severe asphyxic insult $(15,16)$.

\section{TH-Positive Neurons in the SN}

The results of the $\mathrm{TH}$ counts at $\times 5$ magnification are shown in Figure 1, and the corresponding immunohistochemistry photos are shown in Figure 2. The TH neurons were still migrating at the time of sampling, and the differences in the shape of the $\mathrm{SN}$ were observed between the lambs. In some lambs, the $\mathrm{TH}$ neurons had already nearly completed migration into the characteristic line shape of the SN, whereas in others $\mathrm{TH}$ immunoreactivity was found throughout the midbrain (number of lambs with incomplete migration: 1/6 UCO-I, 3/6 UCO-P, 2/5 Sh-I, 3/6 Sh-P, and 1/4 GA). For the purposes of our cell counting, we only counted the TH neurons that fell within the line-shape on four serial slides, on equivalent Bregma levels present in all sheep. Overall, UCO reduced the number of TH-expressing

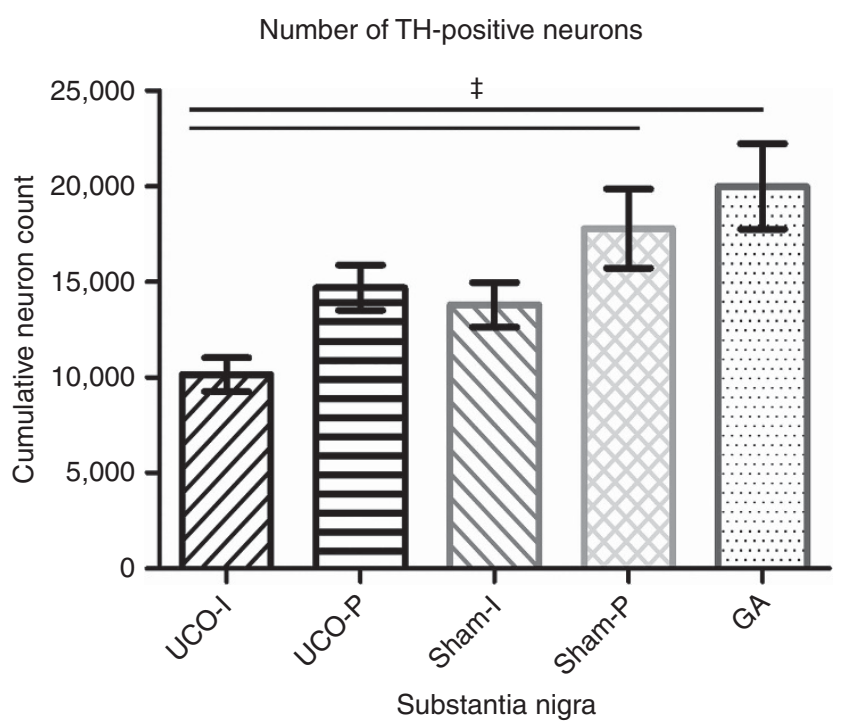

Figure 1. The total TH-neuron counts in the $\mathrm{SN}$ over four slides. The graph shows the mean \pm SEM of the counts $\left({ }^{\ddagger} P<0.01\right)$. SN, substantia nigra; $\mathrm{TH}$, tyrosine hydroxylase. 


\section{Anesthetic use in the near-term HIE lamb Articles}
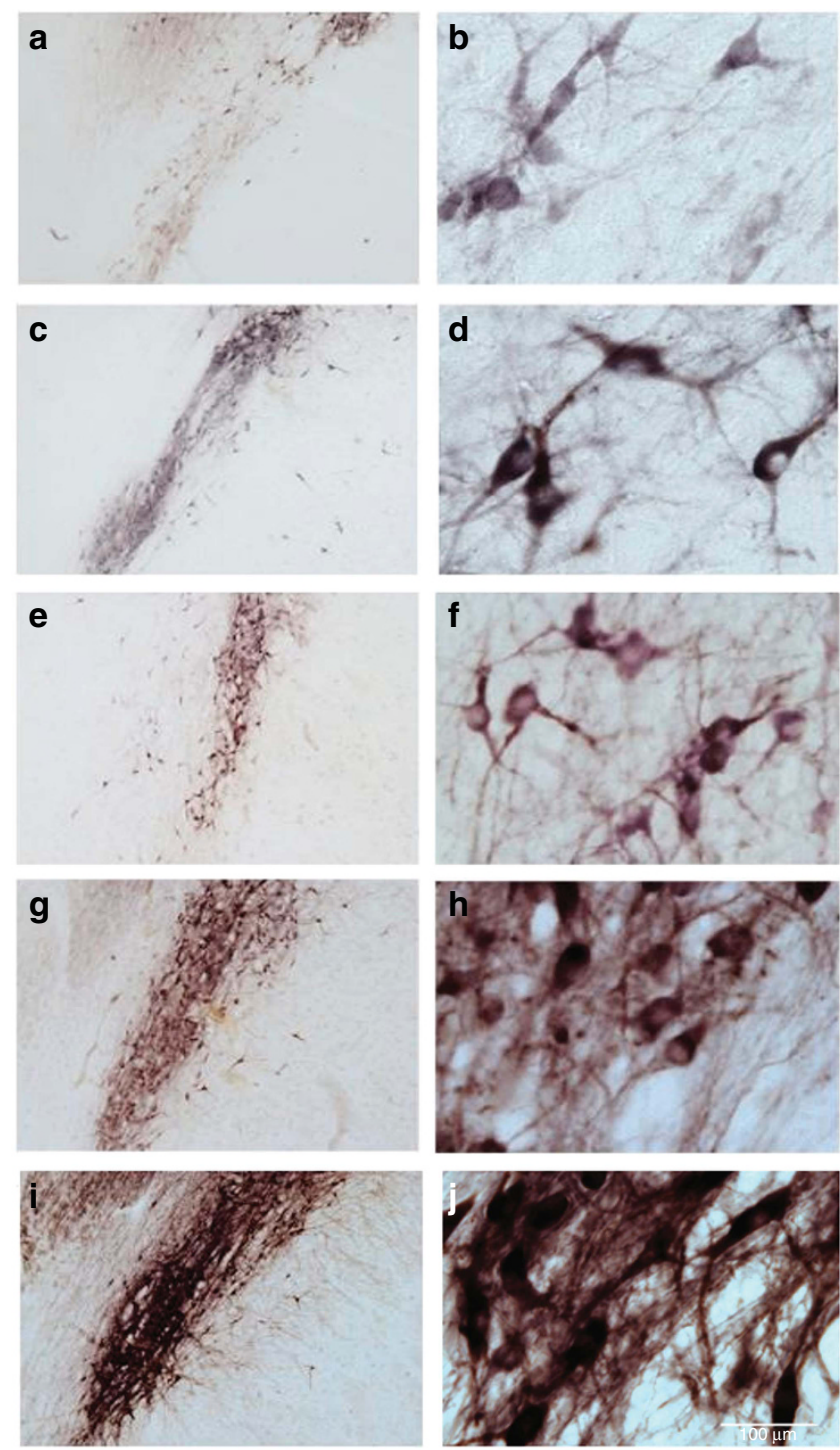

Figure 2. Representative photos of the $\mathrm{TH}$ staining in the $\mathrm{SN}$ at $\times 4$ and $\times 40$ magnification. Bar $=100 \mu \mathrm{m}$. Key: $(\mathbf{a}, \mathbf{b})$ UCO-I, (c,d) UCO-P, (e,f) Sh-l, (g,h) Sh-P, (i,j) GA. SN, substantia nigra; TH, tyrosine hydroxylase; UCO, umbilical cord occlusion.

neurons counted in the SN (UCO-I 10,150 \pm 47 neurons, UCO-P 14,696 \pm 51 neurons, Sh-I 13,806 \pm 51 neurons, Sh-P $17,790 \pm 71$ neurons, GA $19,995 \pm 67$ neurons, combined UCO vs. combined SH; $P<0.05$, UCO-I vs. Sh-P, and UCO-I vs. GA; $P<0.01$ ). Propofol mitigated some of the neuron loss due to UCO. The density of the TH-expressing neurons did not vary significantly between groups (UCO-I $72.35 \pm 4.23$ neurons $/ \mathrm{mm}^{2}$, UCO-P $77.96 \pm 5.69$ neurons $/ \mathrm{mm}^{2}$, Sh-I $76.12 \pm 3.98$ neurons $/ \mathrm{mm}^{2}$, Sh-P $74.55 \pm 4.19$ neurons $/ \mathrm{mm}^{2}$, and GA $113.99 \pm 3.59$ neurons $/ \mathrm{mm}^{2}$ ).

To assess whether the TH-expressing neurons were representative of the number of total neurons in the $\mathrm{SN}$, we assessed the percentage of non-TH-labeled neurons with Nissl staining. Overall, the Sh-I group had the most non-THlabeled neurons $(35.0 \pm 4.5 \%)$, and the GA controls had the fewest $(20.4 \pm 1.8 \%$, Sh-I vs. GA $P<0.001)$. The Sh-P group had $27.1 \pm 5.3 \%$ non-TH-labeled neurons (Sh-P vs. GA and Sh-P vs. Sh-I, $P<0.05)$. Among the UCO-exposed groups, the UCO-I group had more non-TH-labeled neurons $(29.0 \pm 9.8 \%)$ than the UCO-P group $(22.7 \pm 5.4 \%$, UCO-I vs. UCO-P $P<0.05$ ) and the GA group (UCO-I vs. GA $P<0.05)$, as seen in Supplementary Figure S1 Online.

\section{Microglial Response}

The percentage of the total area of interest covered by IBA1positive microglia was assessed in the gray matter of the thalamus and the midbrain area surrounding the $\mathrm{SN}$, in the white matter of the fornix, stratum moleculare below the dentate gyrus (S.M. of the dentate gyrus), and stratum oriens of the CA3 layer (S.O. of the CA3) in the hippocampus. The $\mathrm{SN}$ was defined by the boundaries of $\mathrm{TH}$-positive neurons on adjacent slides (Figure 3 ). In the SN region, there was a trend toward less microglial response in the UCO groups, which did not reach statistical significance (UCO-I 27.75 $\pm 4.17 \%$, UCOP $28.24 \pm 4.37 \%$, Sh-I $33.86 \pm 4.35 \%$, Sh-P $34.92 \pm 4.64 \%$, and GA $34.59 \pm 4.51 \%, P<0.1)$. The percentage differences in IBA1 expression also did not reach statistical significance in the fornix or the S.O. layer in the CA3 region of the hippocampus.

In the thalamus, the UCO-P group had a significantly lower microglial response $(12.14 \pm 3.14 \%)$ than the non-UCOexposed groups (Sh-I 17.54 $\pm 3.39 \%$, Sh-P $16.94 \pm 3.75 \%$, GA $14.71 \pm 2.82 \%$; UCO-P vs. Sh-I $P<0.001$, UCO-P vs. Sh-P, and UCO-P vs. GA $P<0.05)$. The microglial response was also lower than in the UCO-I group $(15.70 \pm 3.59 \%)$, although this was not significant. In the S.M. layer, the microglial response was higher in the non-UCO-exposed groups (Sh-I 33.09 \pm 3.71 , Sh-P $40.71 \pm 4.08 \%$, and GA $26.95 \pm 3.06 \%$ ). This reached significance when comparing the Sh-P group to the UCOexposed groups (UCO-I $30.46 \pm 3.71 \%$, UCO-P 28.36 $\pm 3.65 \%$, Sh-P vs. UCO-I, and Sh-P vs. UCO-P, $P<0.001)$. The microglial response of the thalamus and S.M. is shown in Figure 4.

\section{Tau Phosphorylation}

The CP-13 antibody, against phosphorylated Ser-202-tau, showed region-specific effects in the areas assessed. A direct comparison with the $\mathrm{TH}$ staining of the $\mathrm{SN}$ showed that there was more immunoreactivity in the area adjacent to the SN than in the TH-positive region. This is shown in Figure 3, where the position of the TH-positive neurons on the corresponding slide was largely unstained with the CP-13 antibody, but reactive neurons were observed in the vicinity of the SN. The intensity of the CP-13 staining was overall higher in both the UCO-plus-anesthesia groups (UCO-I 2.12 \pm 0.09 , UCO-P $2.22 \pm 0.14)$ than in the corresponding shamanesthesia groups (Sh-I $1.80 \pm 0.14$, Sh-P $1.97 \pm 0.16$ ), which was higher than the absolute control group (GA $1.31 \pm 0.15$, UCO-I vs. GA, UCO-P vs. GA $P<0.001$, and GA vs. Sh-P $P<0.05)$. 


\section{Articles | Barkhuizen et al.}
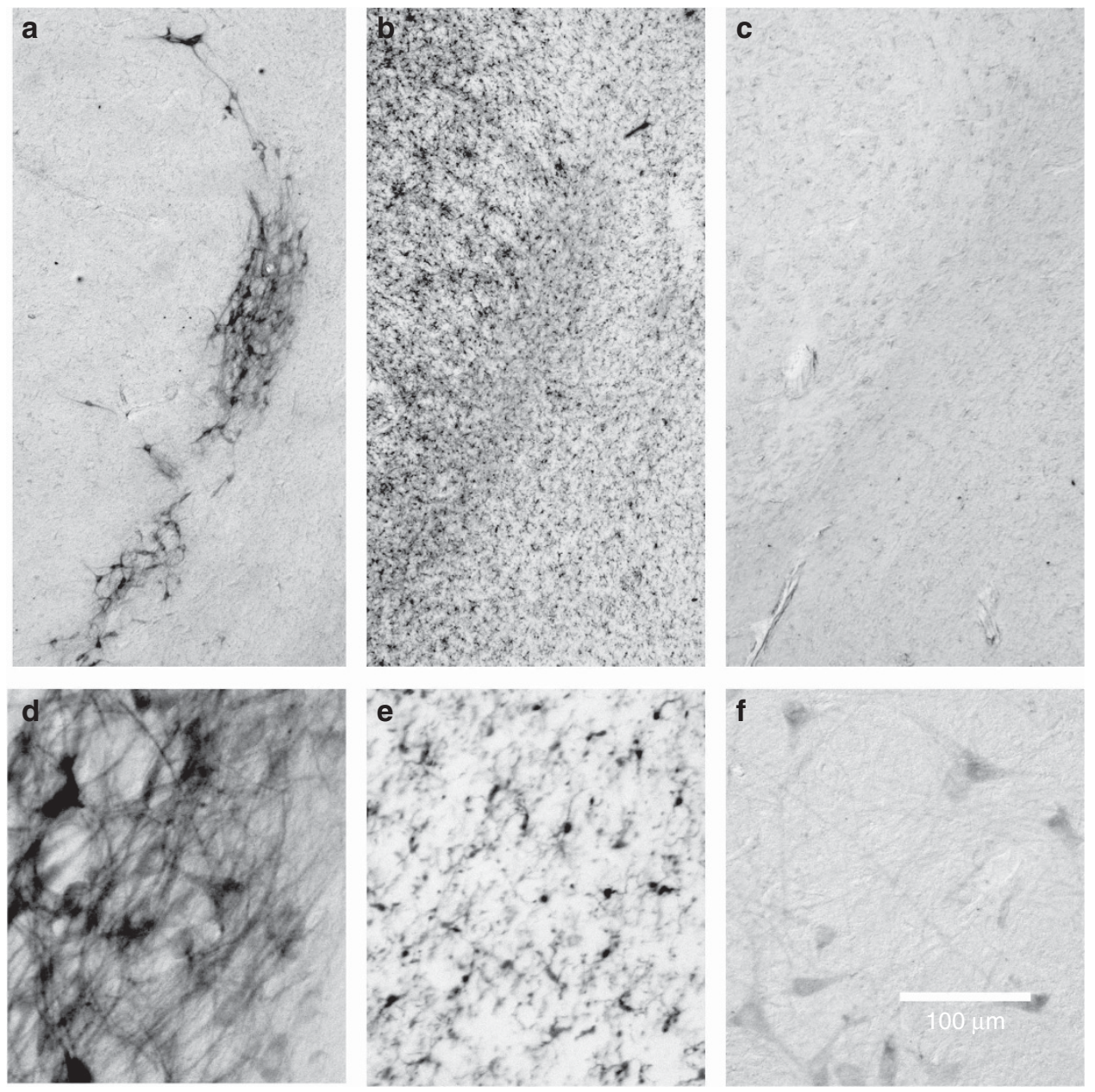

Figure 3. A comparison of the TH (a,d), IBA-1 (b,e), and CP-13 (c, f) immunohistochemistry in the substantia nigra of a UCO-I lamb at $\times 2$ and $\times 10$ magnification. In the IBA-1 (microglia) and the CP-13 (phosphorylated ser-202 tau) images, the dopaminergic neurons of the substantia nigra form an area with a lower reactivity than those of the surrounding midbrain areas. Bar $=100 \mu \mathrm{m}$. UCO, umbilical cord occlusion.

In the thalamus, a similar effect was seen with a pronounced increase in the UCO-P group $(2.53 \pm 0.09)$, in comparison with the other anesthesia-exposed groups (UCOI $2.12 \pm 0.17$, Sh-I $2.22 \pm 0.12$, Sh-P $2.01 \pm 0.09$, UCO-P vs. UCO-I $P<0.001$, and UCO-P vs. Sh-P $P<0.001$ ), and a significant difference between the anesthesia-exposed groups and the GA control group $(1.23 \pm 0.06$, GA vs. all groups, $P<0.0001$; Figure 5). Both UCO and anesthetic usage increased the color intensity of CP-13 staining. The tau-ser202 reactivity in the thalamus predominantly labeled the cell body, as seen in Figure 5.

In the hippocampus, the greatest changes were seen in the $\mathrm{CA} 3$ region, with less reactivity in the Sh-P $(1.85 \pm 0.16)$ and GA $(1.84 \pm 0.22)$ groups than in the UCO- or isofluraneexposed groups (UCO-I $2.38 \pm 0.13$, UCO-P $2.30 \pm 0.11$, Sh-I $2.39 \pm 0.14$, Sh-P vs. Sh-I, Sh-P vs. UCO-I $P<0.05$, Sh-P vs. UCO-P, GA vs. UCO-I, and GA vs. Sh-I, $P<0.1$ ). In dentate gyrus, only the difference between the UCO-P $(2.85 \pm 0.13)$ and GA groups $(2.16 \pm 0.16)$ reached statistical significance $(P<0.05$; Figure 6).
Changes in tau phosphorylation were less pronounced in the other regions tested, with no significant difference between the groups seen in the fornix. The PHF-1 and MC1 antibodies did not detect any phosphorylated Ser396/ Ser404-tau or misfolded tau in our sheep tissue (data not shown). This was likely due to the absence of these pathologies in lambs, as we saw the immunoreactivity in the positive control tissue (Supplementary Figure S2 online).

\section{DISCUSSION}

We investigated whether the choice of anesthetic could limit the loss of TH-expressing neurons in the SN. TH is the ratelimiting step in dopamine synthesis. Studies in rodents suggest that mild HIE increases dopaminergic neurotransmission, whereas severe HIE reduces dopamine transmission. These changes persist in the weeks following the insult (4). Children who had HIE are at increased risk of attention deficits disorders, such as ADHD (2). Structural abnormalities of the SN have been implicated in the pathology of ADHD (6). Given the roles of the $\mathrm{SN}$ in movement disorders, such as 


\section{Anesthetic use in the near-term HIE lamb Articles}
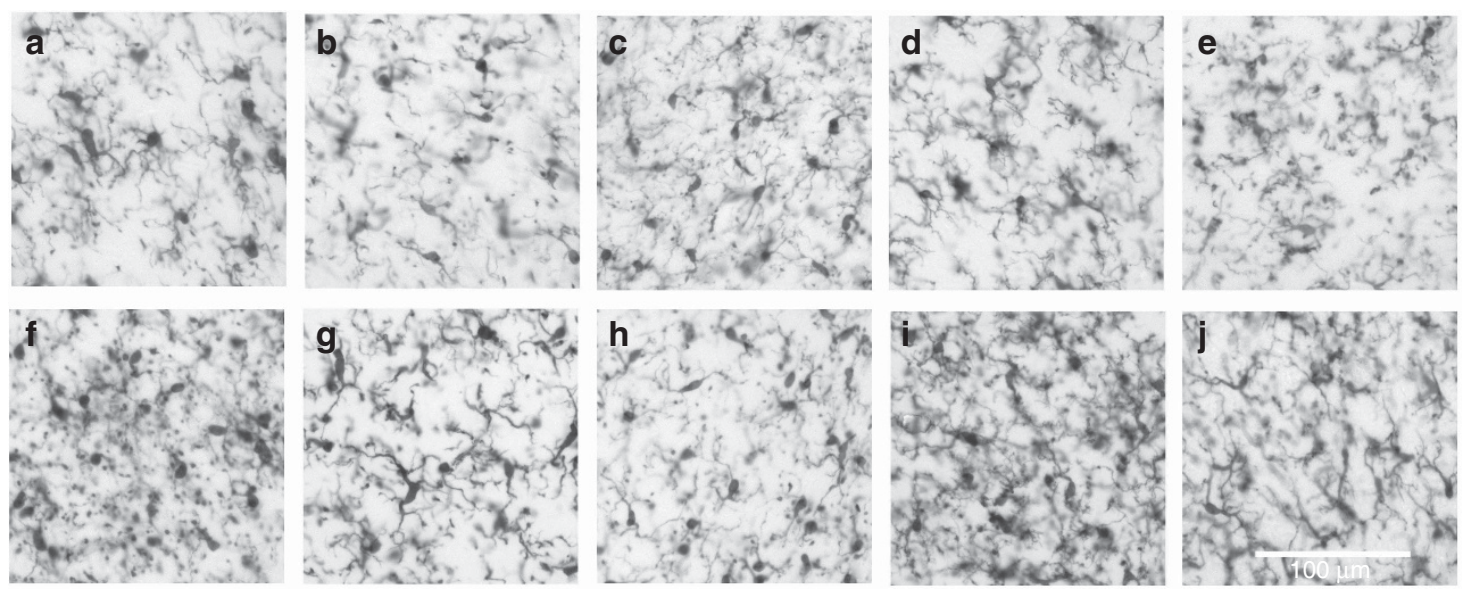

Microglial response in the thalamus and stratum molecular layer of the hippocampus

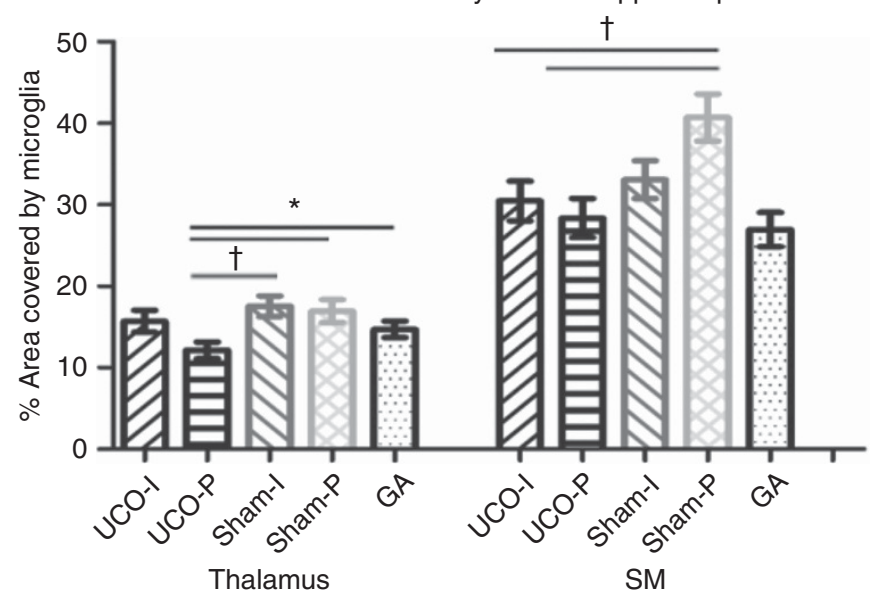

Figure 4. Microglial response in the thalamus and SM layer below the DG of the hippocampus. The graph shows the mean $\pm S E M$ of each group $\left({ }^{*} P<0.05,{ }^{\dagger} P<0.001\right)$. Images were taken at $\times 20$ magnification. Bar $=100 \mu \mathrm{m}$. Key: Thalamus: (a) UCO-I, (b) UCO-P, (c) Sh-I, (d) Sh-P, (e) GA, SM of the DG: (f) UCO-I, (h) UCO-P, (h) Sh-I, (i) Sh-P, (j) GA. DG, dentate gyrus; GA, gestational age; Sh-I, sham isoflurane; Sh-P, sham propofol; SM, stratum molecular; UCO-isoflurane; UCO-P, UCO-propofol; UCO, umbilical cord occlusion.

Parkinson's disease, and attentional deficits, reducing the damage is clinically relevant. We found more dopamineproducing neurons in the $\mathrm{SN}$ of the UCO-P group than in the UCO-I group, and thus the UCO-I combination was more detrimental to the TH-expression phenotype. Propofol anesthesia may also have had a protective role in the $\mathrm{SN}$; however, in the absence of a UCO group without anesthesia, this cannot be established. Because of animal welfare regulations, we could not include this group.

The time course of the $\mathrm{SN}$ development during gestation is not extensively studied in sheep, but, in rats, the first dopamine-positive cells appear on embryonic day 13 (E13). These cells then migrate to the ventral tegmental area on E1516 , and the first neurons migrate to the position of the $\mathrm{SN}$ pars compacta line on E18. At this stage, the dopaminergic SN pars compacta and GABAergic SN pars reticulata are still a single bundle of cells. On E20, this bundle starts separating into the pars compacta and the pars reticulata, with more dopaminergic cells moving to the SN pars compacta on E21 (30). This is the expected day of birth for the rat, when the brain maturity is similar to a human at 28 weeks of gestation. The rat brain resembles the late-preterm human brain, and lamb's brain in this paper, on P7 (4). By this time, the migration of dopaminergic neurons to the $\mathrm{SN}$ pars compacta is essentially complete in the rat, but the dendritic development continues to develop past P14 (30). Our study was only conducted at a single time point $8 \mathrm{~h}$ after the insult, at a GA where the migration of the dopaminergic neurons to the $\mathrm{SN}$ was incomplete in up to $50 \%$ of lambs per group. We further saw differences in the number of remaining $\mathrm{SN}$ neurons, which did not express $\mathrm{TH}$, between the groups. We thus conclude that the anesthetic agents reduced the number of neurons with the dopaminergic neurotransmitter phenotype, but this may not indicate a net loss of cells per se. Although the loss of the neurotransmitter is clinically relevant for the symptoms associated with dopaminergic disturbances, studies with a longer follow-up time need to determine whether the surrounding neurons switch their neurotransmitter phenotype upon recovery to restore dopaminergic homeostasis. 


\section{Articles | Barkhuizen et al.}
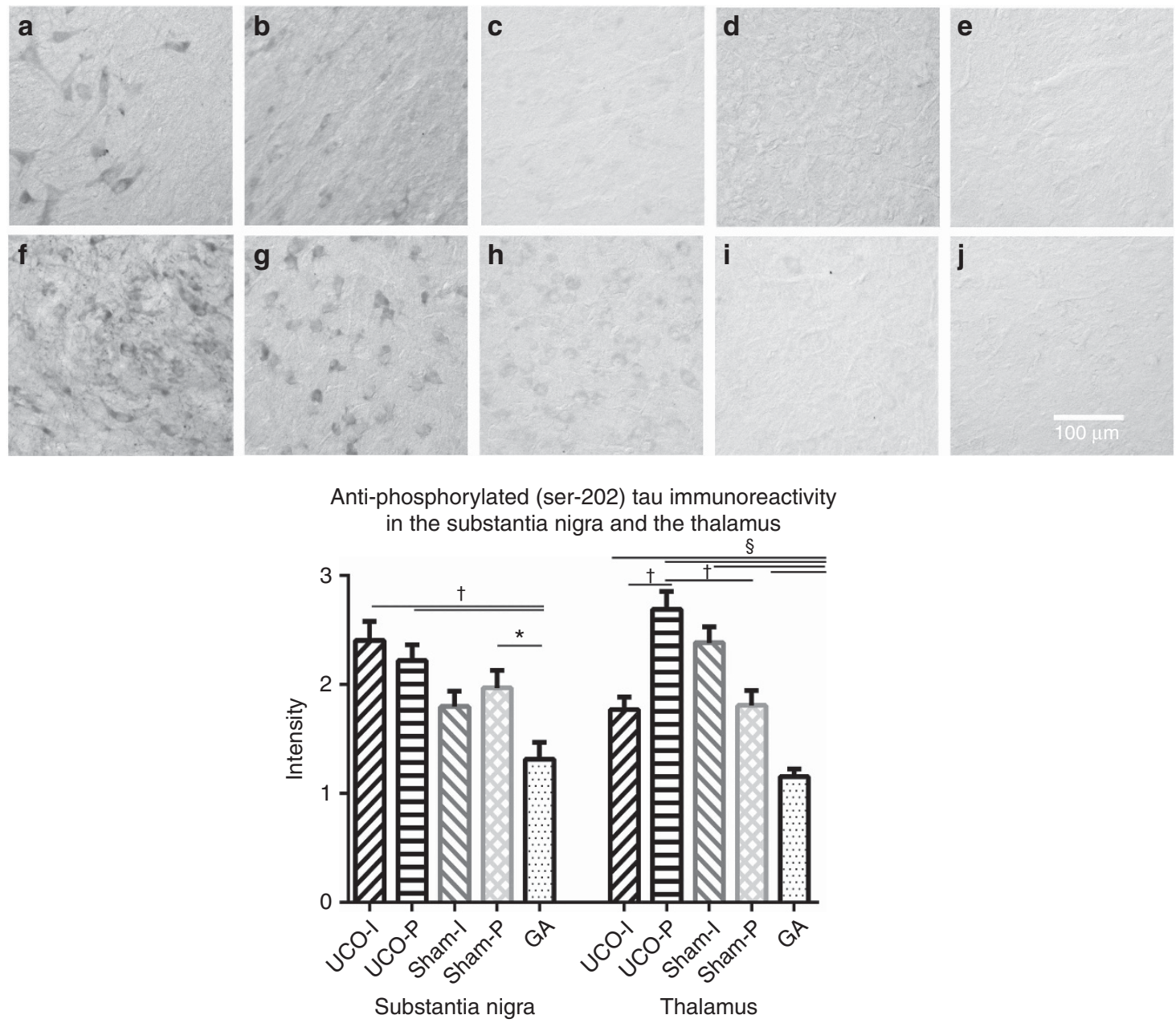

Figure 5. The intensity of anti-phosphorylated (ser-202) tau immunohistochemistry in the $S N$ and the surrounding area, as well as the thalamus. Photographs were taken at $\times 10$ magnification $\left({ }^{\S} P<0.0001,{ }^{\dagger} P<0.001,{ }^{*} P<0.05\right)$. Bar $=100 \mu \mathrm{m}$. Key: SN: (a) UCO-I, (b) UCO-P, (c) Sh-I, (d) Sh-P, (e) GA, Thalamus: (f) UCO-I, (g) UCO-P, (h) Sh-I, (i) Sh-P, (j): GA. GA, gestational age; Sh-I, sham isoflurane; Sh-P, sham propofol; SN, substantia nigra; UCO-isoflurane; UCO-P, UCO-propofol; UCO, umbilical cord occlusion.

We have previously observed the functional and the molecular improvements after UCO with the combined anteand postnatal treatment with anesthetic administration to both the pregnant ewe and newborn lamb (15-17). Here, we investigated whether these anesthetics affected the microglial response. Microglia are the resident innate immune cells in the brain and play a central role in the initiation of an inflammatory response aimed at resolving the injury after HIE. Persistent inflammation may adversely affect brain development (9). Our results did not show a clear effect of the anesthetic choice on the microglial response in the SN region, or other regions studied, and thus presumably these agents activate other neuroprotective pathways not related to neuronal inflammation. Surprisingly, we found lower microglial responses in the UCO-anesthesia groups than in the sham-anesthesia groups, in the thalamus and S.M. layer. This may indicate that anesthetic usage per se is detrimental, but it could limit the microglial response in the injured brain, as reported in earlier studies of focal brain injuries $(12,14)$.
The microglial response initiates around $6 \mathrm{~h}$ after the injury, but only peaks at $48-72 \mathrm{~h}$ (9). Previous UCO studies on fetal sheep (at 70 or $85 \%$ of gestation) found a significant microglial response in the white matter of the hippocampus, cortical, and periventricular regions $(31,32)$. We measured microglial response in both white matter (fornix, S.O. of CA3, and S.M. of the dentate gyrus in the hippocampus) and gray matter in the thalamus and SN region. In preterm infants, white matter injury is more common than gray matter injury, because of the vulnerability of the developing oligodendrocytes. The microglial response partially mediates this. We may not have found an overall increase in microgliosis following UCO since, apart from the hippocampal regions, we focused on the gray matter, and our lambs were killed before the bulk of microglial response.

In humans and sheep, the majority of nervous system development occurs prenatally (33). In the early- and late-preterm infant, the axonal pathways are developing and especially vulnerable to injury (34). Under physiological 


\section{Anesthetic use in the near-term HIE lamb Articles}
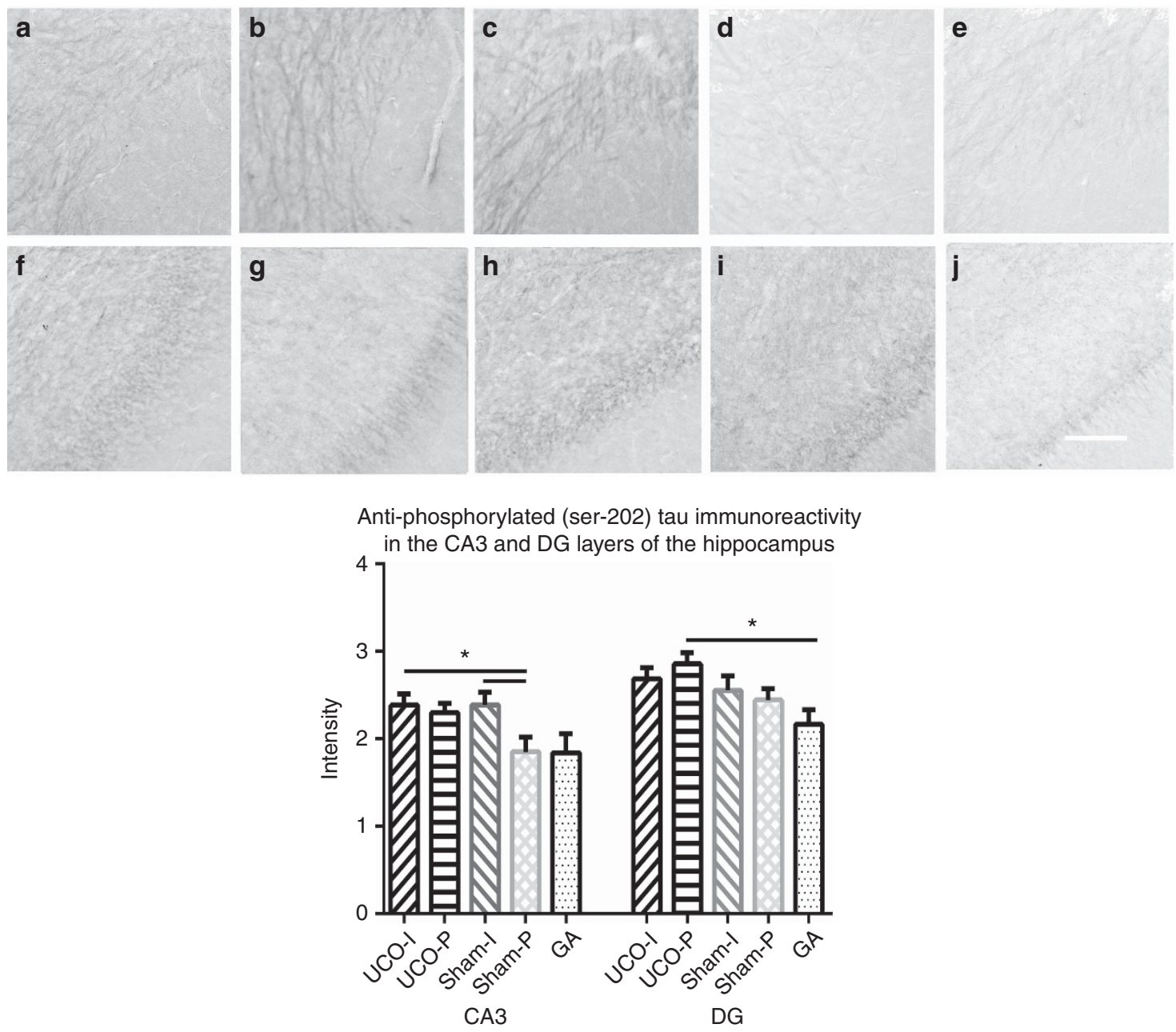

Figure 6. The intensity of anti-phosphorylated (ser-202) tau immunohistochemistry in the hippocampus CA3 and DG layers. The photographs were taken at $\times 10$ magnification $\left({ }^{*} P<0.05\right)$. Bar $=100 \mu \mathrm{m}$. Key: CA3: (a) UCO-I, (b) UCO-P, (c) Sh-I, (d) Sh-P, (e) GA; DG: (f) UCO-I, (g) UCO-P, (h) Sh-I, (i) Sh-P, (j) GA. DG, dentate gyrus; GA, gestational age; Sh-I, sham isoflurane; Sh-P, sham propofol; UCO-isoflurane; UCO-P, UCO-propofol; UCO, umbilical cord occlusion.

conditions, the soluble microtubule-associated protein tau, stabilizes the axonal-microtubule assembly. This is essential for the formation of the neuronal cytoskeleton. Phosphorylation of tau lowers its affinity for the microtubule lattice and thus disrupts its functions $(20,21)$. The disruption of normal tau binding and abnormal tau distribution has been linked to a range of neuropathologies. In the neonatal setting, elevated serum tau is a general predictor of adverse outcomes after encephalopathies (22). Tau is particularly well known for its involvement in late-life neurodegenerative diseases (21). Propofol and other anesthetics have been associated with direct increases in tau phosphorylation, as well as indirect increases mediated by hypothermia. This has been associated with an exacerbated cognitive decline postoperatively in the geriatric population (19).

Hyperphosphorylated tau at residues, such as serine-202, serine-396, and serine-404, form the main component of the paired helical filament neuropathology in Alzheimer's disease, where phosphorylation of ser-202 precedes phosphorylation at ser-396/404 $(35,36)$. Tau is also phosphorylated at these sites during fetal development, which enables the mobility of tau $(20,21)$; however, the extent of phosphorylation at these sites is greater in Alzheimer's disease than in the developing brain (20). In a healthy nervous system, and during development, tau is mainly confined to the axons, but in Alzheimer's disease, the phosphorylated tau is also found in the dendrites and cell bodies $(20,21)$. We found marked phosphorylation in the cell bodies of the groups exposed to anesthesia, with or without additional UCO. This was particularly pronounced in the thalamus (Figure 5). Elevated tau phosphorylation was found in the SN region, thalamus, and hippocampus, indicating a widespread effect of the anesthetics across the brain. We selected additional regions as, in near-term and full-term infants with HIE, the most common site of injury is the central gray matter. The extent of damage to the basal ganglia and thalamus is a predictor of motor impairment after HIE $(28,37)$. The hippocampus is also prone to injury after HIE (27). It is further vulnerable to taumediated neurotoxicity—as seen in Alzheimer's disease-and often affected by anesthesia-induced tau hyperphosphorylation 
(19). We only observed immunoreactivity against early phosphorylation at ser-202 (CP13) in the fetal lambs, but no reactivity against misfolded tau $(\mathrm{MC} 1)$ or the latephosphorylation marker of ser-396/ser-404 (PHF-1). The lack of PHF-1 reactivity likely reflects that only the first indications of tau pathology were present, as this antibody has previously been reported to show neurofibrillary tangle formation in sheep older than 5 years (38), and we validated that the antibodies worked with Alzheimer-related-positive control tissue (Supplementary Figure S2 online). This is encouraging since early hyperphosphorylation may be reversible upon restoration of homeostasis (39), and thus cautions, but does not necessarily limit the use of the anesthetics tested.

\section{CONCLUSION}

In this study, we showed that the choice of anesthetics played a key role in preserving the dopaminergic neurotransmitter phenotype of the SN. The UCO-propofol group had more TH-expressing neurons than the UCO-isoflurane group, although further research is needed to determine whether this signifies propofol-mediated neuroprotection. These findings are clinically relevant, given the susceptibility of the SN to the insult and its role in locomotion and attention deficits. We further noted a possible pathogenic distribution of the phosphorylated tau in this model after exposure to anesthetics, which was aggravated by the insult. This effect could be detrimental if it persists, and long-term studies on this subject are needed.

\section{SUPPLEMENTARY MATERIAL}

Supplementary material is linked to the online version of the paper at http://www.nature.com/pr

\section{ACKNOWLEDGMENTS}

We acknowledge Dr. Peter Davies and the Albert Einstein College of Medicine for the generous gift of the anti-tau antibodies, Dr. Fred van Leeuwen for consulting on the tau staining and the generous gift of the Alzheimer control tissue, and Hellen Steinbusch for her excellent technical assistance. This research was partially supported by the Sistema de Investigación y Desarrollo (SINDE) of the Universidad Católica de Santiago de Guayaquil, Guayaquil, Ecuador, through the grant No SIU- 319: Perinatal asphyxia and stem cell treatment. M. Barkhuizen is funded by the National Research Foundation of South Africa (Grant specific reference number 98217) and the Foundation of Pediatrics, Maastricht University Medical Center + .

Disclosure: The authors declare no conflict of interest.

\section{REFERENCES}

1. Volpe JJ. Brain injury in premature infants: a complex amalgam of destructive and developmental disturbances. Lancet Neurol 2009;8: $110-24$.

2. Van Handel M, Swaab H, De Vries LS, Jongmans MJ. Long-term cognitive and behavioral consequences of neonatal encephalopathy following perinatal asphyxia: a review. Eur J Pediatrics 2007;166: 645-54.

3. Pagida MA, Konstantinidou AE, Tsekoura E, Mangoura D, Patsouris E, Panayotacopoulou MT. Vulnerability of the mesencephalic dopaminergic neurons of the human neonate to prolonged perinatal hypoxia: an immunohistochemical study of tyrosine hydroxylase expression in autopsy material. J Neuropathol Exp Neurol 2013;72:337-50.

4. Barkhuizen M, Van den Hove D, Vles J, Steinbusch H, Kramer B, Gavilanes A. 25 years of research on global asphyxia in the immature rat brain. Neurosci Biobehav Rev 2017;75:166-82.

5. Ezquer ME, Valdez SR, Seltzer AM. Inflammatory responses of the substantia nigra after acute hypoxia in neonatal rats. Exp Neurol 2006;197:391-8.

6. Romanos M, Weise D, Schliesser M, Löffler J, Classen J, Mehler-Wex C. Structural abnormality of the substantia nigra in children with attentiondeficit hyperactivity disorder. J Psychiatr Neurosci 2010;35:55.

7. Davidson JO, Wassink G, van den Heuij LG, Bennet L, Gunn AJ. Therapeutic hypothermia for neonatal hypoxic-ischemic encephalopathywhere to from here? Front Neurol 2015;6:198.

8. Bennet L, Roelfsema V, Pathipati P, Quaedackers J, Gunn A. Relationship between evolving epileptiform activity and delayed loss of mitochondrial activity after asphyxia measured by near-infrared spectroscopy in preterm fetal sheep. J Physiol 2006;572:141-54.

9. Hagberg H, Gressens P, Mallard C. Inflammation during fetal and neonatal life: implications for neurologic and neuropsychiatric disease in children and adults. Ann Neurol 2012;71:444-57.

10. Rudolph U, Antkowiak B. Molecular and neuronal substrates for general anaesthetics. Nat Rev Neurosci 2004;5:709-20.

11. Sinner B, Becke K, Engelhard K. General anaesthetics and the developing brain: an overview. Anaesthesia 2014;69:1009-22.

12. Archer DP, Walker AM, McCann SK, Moser JJ, Appireddy RM. Anesthetic neuroprotection in experimental stroke in rodentsa systematic review and meta-analysis. J Am Soc Anesthesiol 2017;126: 653-65.

13. Ye X, Lian Q, Eckenhoff MF, Eckenhoff RG, Pan JZ. Differential general anesthetic effects on microglial cytokine expression. PLoS ONE 2013;8: e52887.

14. Luo T, Wu J, Kabadi SV, et al. Propofol limits microglial activation after experimental brain trauma through inhibition of nicotinamide adenine dinucleotide phosphate oxidase. Anesthesiology 2013;119:1370-88.

15. Seehase M, Houthuizen P, Jellema RK, et al. Propofol administration to the fetal-maternal unit reduces cardiac injury in late-preterm lambs subjected to severe prenatal asphyxia and cardiac arrest. Pediatr Res 2013;73:427-34.

16. Seehase M, Jennekens W, Zwanenburg A, et al. Propofol administration to the maternal-fetal unit improved fetal EEG and influenced cerebral apoptotic pathway in preterm lambs suffering from severe asphyxia. Mol Cell Pediatr 2015;2:4.

17. Smit AL, Seehase M, Stokroos RJ, et al. Functional impairment of the auditory pathway after perinatal asphyxia and the short-term effect of perinatal propofol anesthesia in lambs. Pediatr Res 2013;74:34-8.

18. Zakowski MI, Herman NL The placenta: anatomy, physiology, and transfer of drugs. In: Chestnut's Obstetric Anesthesia: Principles and Practice E-Book. 3rd edn. Chestnut DH (ed). Philadelphia: Mosby Inc, 2004, 60.

19. Whittington RA, Bretteville A, Dickler MF, Planel E. Anesthesia and tau pathology. Progr Neuro-Psychopharmacol Biol Psychiatry 2013;47: $147-55$.

20. Yu Y, Run X, Liang Z, et al. Developmental regulation of tau phosphorylation, tau kinases, and tau phosphatases. J Neurochem 2009;108:1480-94.

21. Spillantini MG, Goedert M. Tau pathology and neurodegeneration. Lancet Neurol 2013;12:609-22.

22. Takahashi K, Hasegawa S, Maeba S, et al. Serum tau protein level serves as a predictive factor for neurological prognosis in neonatal asphyxia. Brain Dev 2014;36:670-5.

23. Wu H, Li Z, Yang X, Liu J, Wang W, Liu G. SBDPs and Tau proteins for diagnosis and hypothermia therapy in neonatal hypoxic ischemic encephalopathy. Exp Ther Med 2017;13:225-9.

24. Strackx E, Van den Hove D, Steinbusch H, et al. A combined behavioral and morphological study on the effects of fetal asphyxia on the 
nigrostriatal dopaminergic system in adult rats. Exp Neurol 2008;211: 413-22.

25. Irwin DJ, VM-Y Lee, Trojanowski JQ. Parkinson's disease dementia: convergence of [alpha]-synuclein, tau and amyloid-[beta] pathologies. Nat Rev Neurosci 2013;14:626-36.

26. Heneka MT, Kummer MP, Latz E. Innate immune activation in neurodegenerative disease. Nat Rev Immunol 2014;14:463.

27. Volpe JJ. The neurological outcome of perinatal asphyxia. Early Brain Damage 2012: 151.

28. Counsell SJ, Tranter SL, Rutherford MA Magnetic resonance imaging of brain injury in the high-risk term infant. Seminars in Perinatology. Elsevier, 2010:67-78.

29. Zhou M, Huang T, Collins N, et al. APOE4 induces site-specific tau phosphorylation through calpain-CDK5 signaling pathway in EFADTg mice. Current Alzheim Res 2016;13:1048-55.

30. Voorn P, Kalsbeek A, Jorritsma-Byham B, Groenewegen H. The pre-and postnatal development of the dopaminergic cell groups in the ventral mesencephalon and the dopaminergic innervation of the striatum of the rat. Neuroscience 1988;25:857-7.

31. Jellema RK, Lima Passos V, Zwanenburg A, et al. Cerebral inflammation and mobilization of the peripheral immune system following global hypoxia-ischemia in preterm sheep. J Neuroinflammation 2013;10.1:807.

32. Davidson JO, Yuill CA, Zhang FG, Wassink G, Bennet L, Gunn AJ. Extending the duration of hypothermia does not further improve white matter protection after ischemia in term-equivalent fetal sheep. Sci Rep 2016;6:25178.

33. van den Heuij LG, Wassink G, Gunn AJ, Bennet L. Using pregnant sheep to model developmental brain damage. Prenatal Postnatal Determinants Dev 2016: 327-41.

34. Kostović I, Jovanov-Milošević N. The development of cerebral connections during the first $20-45$ weeks' gestation. Seminars in Fetal and Neonatal Medicine. Fellman V, Hellström-Westas L, Rosén I (eds). Elsevier, New York, NY, 2006, pp 415-22.

35. Su JH, Cummings BJ, Cotman CW. Early phosphorylation of tau in Alzheimer's disease occurs at Ser-202 and is preferentially located within neurites. Neuroreport 1994;5:2358-62.

36. Spires TL, Orne JD, SantaCruz K, et al. Region-specific dissociation of neuronal loss and neurofibrillary pathology in a mouse model of tauopathy. Am J Pathol 2006;168:1598-607.

37. Cabaj A, Bekiesińska-Figatowska M, Mądzik J. MRI patterns of hypoxicischemic brain injury in preterm and full term infants-classical and less common MR findings. Pol J Radiol 2012;77:71-6.

38. Nelson PT, Greenberg SG, Saper CB. Neurofibrillary tangles in the cerebral cortex of sheep. Neurosci Lett 1994;170:187-90.

39. Van der Harg J, Nölle A, Zwart R, et al. The unfolded protein response mediates reversible tau phosphorylation induced by metabolic stress. Cell Death Dis 2014;5:e1393. 